\title{
Associations Between Fine Particulate Matter (PM2.5) and Childhood-Onset Systemic Lupus Erythematosus
}

\author{
Chen-Hao Mai ${ }^{1,2} \cdot$ Yen-Ju Shih ${ }^{3} \cdot$ Cheng-Li Lin ${ }^{4,5} \cdot$ Chang-Ching Wei ${ }^{1,6}$
}

Received: 23 June 2021 / Accepted: 22 October 2021/Published online: 18 November 2021

(c) Dr. K C Chaudhuri Foundation 2021

To the Editor: Although it is not clearly known what factors play a role in the pathogenesis of childhood-onset systemic lupus erythematosus (cSLE), it has been reported that exposure to $\mathrm{SO}_{2}$ and $\mathrm{O}_{3}$ lead to an increase in pediatric rheumatic diseases hospitalizations, and exposure to $\mathrm{PM}_{10}, \mathrm{NO}_{2}$, and CO may increase the risk of disease activity in cSLE $[1,2]$. Recently, a study from Brazil demonstrated that short-term exposure to both indoor and outdoor $\mathrm{PM}_{2.5}$ was associated with increases in airway inflammation and systemic inflammation in cSLE patients [3]. However, these studies only assess the exposure to $\mathrm{PM}_{2.5}$ and the disease activity and hospitalization over a short period of time. There are limited studies examining the association between $\mathrm{PM}_{2.5}$ variation and the incidence of cSLE over a long period of time. Therefore, our objective was to evaluate the effects of air pollution on the risk of developing cSLE in Taiwan from 2000 to 2012.

Chen-Hao Mai and Yen-Ju Shih contributed equally to this work.

This research was presented as a poster at the 2019 American College of Rheumatology (ACR)/Association of Rheumatology Professionals (ARP) Annual Meeting (Abstract No. 670).

Chang-Ching Wei

weilonger@gmail.com

1 Department of Pediatric Allergy Immunology and Rheumatology, Children's Hospital, China Medical University Hospital, Taichung 40447, Taiwan

2 Department of Pediatrics, Chang Bing Show Chwan Memorial Hospital, Changhua, Taiwan

3 Rheumatology and Immunology Center, Department of Internal Medicine, China Medical University Hospital, Taichung, Taiwan

4 Management Office for Health Data, China Medical University Hospital, Taichung, Taiwan

5 Institute of Biostatistics, China Medical University, Taichung, Taiwan

6 School of Medicine, China Medical University, Taichung, Taiwan
This is the first large population study to evaluate the exposure of ambient $\mathrm{PM}_{2.5}$ and the occurrence of cSLE over a long period of time. A total of 394 children $(0.16 \%)$ were newly diagnosed with cSLE during the observation period. The incidence rate for cSLE increased with $\mathrm{PM}_{2.5}$ levels, from $4.7(\mathrm{Q} 1)$ to $21.9(\mathrm{Q} 4)$ per 100,000 person-years. In the multivariable Cox proportional hazard regression, the adjusted HR for SLE increased with the $\mathrm{PM}_{2.5}$ exposure concentrations from 2.74 to 4.23 compared with that for those exposed to the corresponding concentrations in the Q1 level.

This longitudinal study provides evidence that long-term variations in $\mathrm{PM}_{2.5}$ levels are risk factors for the development of cSLE. Although further studies are required to confirm these associations, our study suggests that awareness, education, and appropriate public policy for better air quality will result in a lower incidence of cSLE and will improve public health.

Funding This study is supported in part by China Medical University Hospital (CRS-108-015, DMR-HHC-109-9, DMR-HHC-110-7, and DMR-108-200).

\section{Declarations}

Conflict of Interest None.

\section{References}

1. Vidotto JP, Pereira LA, Braga AL, et al. Atmospheric pollution: influence on hospital admissions in paediatric rheumatic diseases. Lupus. 2012;21:526-33.

2. Fernandes EC, Silva CA, Braga AL, Sallum AM, Campos LM, Farhat SC. Exposure to air pollutants and disease activity in juvenile-onset systemic lupus erythematosus patients. Arthritis Care Res (Hoboken). 2015;67:1609-14.

3. Alves AGF, de Azevedo Giacomin MF, Braga ALF, et al. Influence of air pollution on airway inflammation and disease activity in childhood-systemic lupus erythematosus. Clin Rheumatol. 2018;37:683-90.

Publisher's Note Springer Nature remains neutral with regard to jurisdictional claims in published maps and institutional affiliations. 